\title{
Fermentation Products of
} Paenibacillus bovis sp. nov. BD3526 Alleviates the Symptoms of Type 2 Diabetes Mellitus in GK Rats

\begin{abstract}
Zhenyi Qiao ${ }^{1 \dagger}$, Jin Han ${ }^{1,2 t}$, Huafeng Feng ${ }^{1}$, Huajun Zheng ${ }^{3,4}$, Jiang Wu ${ }^{1}$, Caixia Gao', Meng Yang ${ }^{1,5}$, Chunping You ${ }^{1}$, Zhenmin Liu ${ }^{1}$ and Zhengjun $\mathrm{Wu}^{1 *}$

1 State Key Laboratory of Dairy Biotechnology, Shanghai Engineering Research Center of Dairy Biotechnology, Dairy Research Institute, Bright Dairy \& Food Co., Ltd., Shanghai, China, ${ }^{2}$ College of Food Science and Technology, Shanghai Ocean University, Shanghai, China, ${ }^{3}$ Shanghai-MOST Key Laboratory of Health and Disease Genomics, Chinese National Human Genome Center at Shanghai, Shanghai, China, ${ }^{4}$ Key Laboratory of Reproduction Regulation of NPFPC, Shanghai Institute of Planned Parenthood Research, IRD, Fudan University, Shanghai, China, ${ }^{5}$ School of Life Sciences, Shanghai University, Shanghai, China
\end{abstract}

Gut microbiota is closely related to type 2 diabetes mellitus (T2DM). The gut microbiota of patients with T2DM is significantly different from that of healthy subjects in terms of bacterial composition and diversity. Here, we used the fermentation products of Paenibacillus bovis sp. nov. BD3526 to study the disease progression of T2DM in Goto-kakisaki (GK) rats. We found that the symptoms in GK rats fed the fermentation products of BD3526 were significantly improved. The 16S rRNA sequencing showed that the fermentation products of BD3526 had strong effects on the gut microbiota by increasing the content of Akkermansia. In addition, the interaction of the genus in the gut of the BD3526 group also significantly changed. Additional cytokine detection revealed that the fermentation products of BD3526 can reduce the inflammatory factors in the intestinal mucus of GK rats and thereby inhibit the inflammatory response and ameliorate the symptoms of T2DM.

Keywords: type 2 diabetes, gut microbiota, Paenibacillus bovis sp. nov. BD3526, Akkermansia muciniphila, cytokine

\section{INTRODUCTION}

According to data published by the International Diabetes Federation, there were 425 million people with diabetes worldwide in 2017. Type 2 diabetes mellitus (T2DM) is characterized by a sustained decrease in the insulin secretion of pancreatic $\beta$-cells, which leads to insufficient insulin to fulfill the requirement of the body. Long-term T2DM in the human body can cause serious complications, for example in the kinds of kidney disease, cancer and cardiovascular disease (Stern, 1995; Coughlin et al., 2004).

In the last two decades, evidence has accumulated that the pathogenesis of T2DM and its complications may be related to inflammation factors (i.e., IL-1 $\beta$, IL-6, MCP-1, TNF- $\alpha$ and IFN$\gamma$ ) (Cuman et al., 2001; Rotter et al., 2002; Masters et al., 2010; Westwellroper et al., 2011; WestwellRoper et al., 2014; Greer et al., 2016). For example, IL-6 is the most endocrine cytokine and is not only produced by immunocompetent cells but also by fat cells involved in the body's inflammatory response and energy metabolism (Rotter et al., 2002). In patients with inflammation, the IL- 6 levels are elevated. Excess IL- 6 promotes pancreatic islet $\beta$ lymphocyte differentiation and overexpresses the IgG gene, which promotes excessive activation of T lymphocytes and thereby 
causes destruction and death of islet $\beta$ cells (André-Schmutz et al., 2010; Donath, 2013). Therefore, the therapeutic targets for T2DM have shifted from simple hypoglycemic drugs (e.g., insulin and acarbose) to drugs suppressing inflammatory factors (such as IL-1 receptors blockade Anakinra and IKK $\beta$-NF-KB inhibition Salsalate) (Donath, 2013).

The gut microbiota plays an essential role in the development of T2DM by skewing the host immune response to the inflammatory reaction (Karlsson et al., 2013). Wu et al. (2017) found that clinical patients with T2DM had significant changes in the composition and diversity of the gut microbiota after taking metformin being beneficial to improve the symptoms of T2DM. Some sources claim that Akkermansia muciniphila in the feces is significantly enriched in patients and mice with T2DM after taking metformin (Karlsson et al., 2013; Lee and Ko, 2014; Shin et al., 2014; Zhang et al., 2015; de la Cuesta-Zuluaga et al., 2017; Forslund et al., 2017). Low levels of A. muciniphila in the intestine may correlate with the thinning of the mucosal layer, which leads to a weakened intestinal barrier function (Derrien et al., 2004). In addition, A. muciniphila was reduced in patients with obesity and T2DM (Derrien et al., 2004; Everard et al., 2013). Further out, a recent study also claims that $A$. muciniphila can improve the response rate of tumor patients to PD-1/PD-L1 immunotherapy (Routy et al., 2018).

We have since isolated a novel bacterium designated as Paenibacillus bovis sp. nov. BD3526 from Tibetan yak milk in a previous work (Hang et al., 2016). This strain can synthesize exopolysaccharides with immunomodulatory activity in vitro (Xu et al., 2016). Instead, attempts to identify the relationship between exopolysaccharides and T2DM are not yet resolved. In this work, the lyophilized supernatant of the fermented milk by the BD3526 strain was fed to GK rats and its effect on the diabetic phenotype of the rats was observed. It was found that the symptoms of diabetes in the rats fed the supernatant were significantly improved. The content of A. muciniphila in the intestines was significantly increased, which coincided with a decrease in the inflammatory response in the intestine.

\section{MATERIALS AND METHODS}

\section{Bacterial Strain and Cultivation}

Paenibacillus bovis sp. nov BD3526 (CGMCC $8333=$ DSM28815 = ATCC BAA-2746) was provided by the State Key Laboratory of Dairy Biotechnology, Shanghai 200436, China. The bacterial strain was routinely cultivated aerobically on milk agar at $30^{\circ} \mathrm{C}$ for $24 \mathrm{~h}$. The medium was prepared by adding $10 \mathrm{~mL}$ sterile $10 \%(\mathrm{w} / \mathrm{w})$ reconstituted skim milk to $100 \mathrm{~mL}$ melted $1.5 \%(\mathrm{v} / \mathrm{w})$ agar solution. The strain was stored in sterile $10 \%(\mathrm{w} / \mathrm{w})$ reconstituted skim milk supplement with $10 \%(\mathrm{v} / \mathrm{v})$ glycerol at $-80^{\circ} \mathrm{C}$.

\section{Preparation of the BD3526 Strain Fermentation Products}

A loop of freshly cultivated BD3526 on milk agar was inoculated into a $100-\mathrm{mL}$ flask containing $20 \mathrm{~mL}$ sterile $10 \%(\mathrm{w} / \mathrm{w})$ reconstituted skim milk and cultivated at $30^{\circ} \mathrm{C}$ at $180 \mathrm{rpm}$ for $24 \mathrm{~h}$. The culture was then transferred to a 250 -mL flask containing $50 \mathrm{~mL}$ sterile $10 \%(\mathrm{w} / \mathrm{w})$ reconstituted milk at a ratio of $4 \%(\mathrm{v} / \mathrm{v})$ and cultivated at the same conditions as mentioned above for $72 \mathrm{~h}$. Samples at different intervals were boiled for $5 \mathrm{~min}$ and then centrifuged at $12000 \times \mathrm{g}$ at $4^{\circ} \mathrm{C}$ for $20 \mathrm{~min}$. The supernatant was neutralized with $1 \mathrm{M} \mathrm{NaOH}$ to $\mathrm{pH} 6.8$ and assayed for the inhibitory activity to alpha glucosidase (EC 3.2.1.20) utilizing $p$-nitrophenyl a-D-glucopyranoside (PNPG) as the substrate. The $72 \mathrm{~h}$ culture with an inhibitory activity to alpha glucosidase of approximately $60 \%$ (data unpublished) was boiled and centrifuged at the same condition mentioned above. The supernatant was lyophilized under a vacuum. The lyophilized powder was then cold stored. Before gavage of the experimental animals, the powder was redissolved in distilled water at a concentration of $50 \mathrm{mg} / \mathrm{mL}$.

\section{Animal Experiments}

A total of 10 twelve-week-old Goto-kakisaki (GK) rats were adopted for 1 week and then randomly divided into two groups. The rats in the BD3526 group were gavaged daily with $2 \mathrm{~mL}$ $50 \mathrm{mg} / \mathrm{mL}$ lyophilized fermentation products of BD3526, whereas the rats in the control group were gavaged with $2 \mathrm{ml} 50 \mathrm{mg} / \mathrm{mL}$ skim milk powder. The animals were individually caged with free access to a normal chewing bar and drinking water. On the day of the assay of postprandial blood glucose, the animals were first fed a normal chewing bar for $1 \mathrm{~h}$ and then gavage was conducted. After the gavage, the chewing bars were removed from the cage and a blood sample from the tail was collected $2 \mathrm{~h}$ after the gavage and analyzed with commercial blood glucose test strips (Sannuo, Shenzhen, China).

The postprandial blood glucose and body weight were measured weekly and fecal samples were collected. Glycated hemoglobin was measured at week 5. From the 6th week and thereafter, the gavage was terminated and the animals were restored to normal management. Postprandial blood glucose and body weight was continuously measured (weekly) and fecal samples were collected until the 9th week. After the 9th week, the intestinal mucus of the rats was collected for detection of the immunological factor. Feeding of the rates was conducted at Shanghai Slac Experimental Animal Co., Ltd.

\section{DNA Extraction and PCR Amplification}

Microbial DNA was extracted from the fecal samples using the E.Z.N.A. ${ }^{\circledR}$ stool DNA Kit (Omega Biotek, Norcross, GA, United States), according to the manufacturer's protocols. The final DNA concentration and purification were determined by a NanoDrop 2000 UV-vis spectrophotometer (Thermo Scientific, Wilmington, DE, United States), and the DNA quality was checked by $1 \%$ agarose gel electrophoresis. The V3-V4 hypervariable regions of the bacteria $16 \mathrm{~S}$ rRNA gene were amplified by a thermocycler PCR system (GeneAmp 9700, ABI, United States). The PCRs were performed in triplicate with the $20-\mu \mathrm{L}$ mixture containing $4 \mu \mathrm{L} 5 \times$ FastPfu Buffer, $2 \mu \mathrm{L} 2.5 \mathrm{mM}$ dNTPs, $0.8 \mu \mathrm{L}$ of each primer $(5 \mu \mathrm{M}), 0.4 \mu \mathrm{L}$ FastPfu Polymerase and $10 \mathrm{ng}$ template DNA. The resulting PCR products were extracted from a $2 \%$ agarose gel, further purified using the AxyPrep DNA Gel Extraction Kit (Axygen Biosciences, Union 
City, CA, United States) and quantified using QuantiFluor TMST (Promega, United States), according to the manufacturer's protocol.

\section{High-Throughput Sequencing}

The purified amplicons were pooled in equimolar and pairedend sequenced $(2 \times 300 \mathrm{bp})$ on an Illumina MiSeq platform (Illumina, San Diego, CA, United States), according to the standard protocols by Sinotech Genome Technology Co., Ltd. (Shanghai, China).

\section{Bioinformatic Analysis and Statistical Analysis}

In the bioinformatics analysis of $16 \mathrm{~S}$ rRNA sequencing samples, we used an online cloud platform from Sinotech Genome Technology Co., Ltd.

Specifically, we used Usearch (version 7.0) ${ }^{1}$ to perform cluster analysis on the OTUs. The diversity of the $\mathrm{BD} 3526$ group and the control group was analyzed using mother software (version v.1.30.1 $)^{2}$. Furthermore, we also used the PLS-DA analysis in the $\mathrm{R}$ language mixOmics package and created a distance map for each sample.

In the comparison of different strains, we used metagenome seq derived from the $\mathrm{R}$ language package to perform the Zero-inflated Gaussian distribution to process the impact of sequencing depth, and we finally found a difference based on the linear model.

In the random forest analysis, we used the Random Forest package and the plotROC package to quickly and efficiently select the species category that is most important for sample classification and to perform relevant ROC verification.

We used Networkx software ${ }^{3}$ in the collinear network analysis and correlation network analysis.

The $16 \mathrm{~S}$ function prediction is used to standardize the OTU abundance table by PICRUSt (the PICRUSt software stores the $\mathrm{KO}$ information corresponding to the greengene id), that is, to remove the influence of the number of copies of the $16 \mathrm{~S}$ marker gene in the species genome the. We then pass the greengene id corresponding to each out, obtain the KEGG Ortholog (KO) information corresponding to the out, and calculate the $\mathrm{KO}$ abundance. According to the KEGG database information, the KO, Pathway, and EC information can be obtained, and the abundance of each functional category can be calculated based on OTU abundance. In addition, for the Pathway, PICRUSt can be used to obtain three levels of information on the metabolic pathways and to obtain abundance tables for each level.

In addition, we also used Graphpad Prism6 software to create statistical images of the statistical data.

\section{In vitro Gut Simulator}

The basic protocols were referred to the published article ( $\mathrm{Wu}$ et al., 2017). Briefly, $2 \%$ fecal samples were gathered and cultured in $5 \mathrm{~mL} \mathrm{BHI}$ broth for $3 \mathrm{~h}$ at $37^{\circ} \mathrm{C}$ and the $2 \%$ preculture was

\footnotetext{
${ }^{1}$ http://drive5.com/uparse/

${ }^{2}$ http://www.mothur.org/wiki/Schloss_SOP\#Alpha_diversity

${ }^{3} \mathrm{http} / / /$ networkx.github.io
}

seeded into the feed medium. The medium was composed by: (in g/liter) arabinogalactan (1.0), pectin (2.0), xylose (1.5), starch (3.0), glucose (0.4), yeast extract (3.0), peptone (1.0), mucin type II (4.0), and cysteine (0.5). The medium was acidified to around $\mathrm{pH} 2$ with $6-\mathrm{M} \mathrm{HCl}$ to simulate digestion processes, and neutralized with simulated pancreatic juice to a $\mathrm{pH}$ of around 6.9. The simulated pancreatic juice contained: (in g/liter) $\mathrm{NaHCO}_{3}$ (12.5), Oxgall bile salts (6.0), and pancreatin (0.9). Unless the experiment performed for 3 days, all the cultures were under the condition of anaerobic.

\section{Cytokine Detection}

The protocol of cytokine detection can be referred to from the instructions of a rat Cytokine Antibody Array Kit (Abcam, ab133992). Briefly, the membrane was incubated with blocking buffer. Then, $1 \mathrm{~mL}$ large intestine mucus was added to bind the antibodies that are fixed at the surface of the membrane. Following this step, the membrane was washed with washing buffer five times and then incubated with $1 \mathrm{X}$ biotin-conjugated anti-cytokines and 1X HRP-conjugated streptavidin. Finally, the membrane was exposed by X-ray film.

\section{Accession Numbers}

The Paenibacillus bovis sp. nov. BD3526 mentioned in this article can be found in the ATCC database. The ATCC number of Paenibacillus bovis sp. nov. BD3526 is BAA-2746. Highthroughput sequencing data from this study was deposited in the Sequence Read Archive (SRA) databases under the following accession number: SRP151163 and PRJNA508215.

\section{Ethics Statement}

The project use and care of the animals in this research was reviewed and approved by the Shanghai Laboratory Animal Management Office (SYXK [Shanghai] 2017-0008).

The animals used in the research were utilized based on appropriate experimental procedures. All of the animals were lawfully acquired, and their retention and use were in compliance with federal, state and local laws and regulations in every case and in accordance with the Institutional Animal Care and Use Committee of SLAC (IACUC) Guide for Care and Use of Laboratory Animals.

Animals used in this research received every consideration for their comfort and were properly housed, fed, and their surroundings kept in a sanitary condition.

The use of animals was in accordance with the IACUC Guide for Care and Use of Laboratory Animals. A minimal number of mice were used during the experiments. Appropriate anesthetics were used to eliminate sensibility to pain during all of the surgical procedures.

\section{RESULTS}

\section{Diabetic Symptoms Were Alleviated in the BD3526 Group}

In our previous work, we found that the BD3526 strain could synthesize a large amount of exopolysaccharides (36.25 g/L) 
with in vitro immunomodulatory activity (Xu et al., 2016), which might also play roles in retarding the development of diabetes in vivo. Besides EPS, monosaccharides, for example in the shapes of fructose, could also be identified in the fermentation products of BD3526. Therefore, to observe the effect of the BD3526 strain fermentation products in skim milk on blood glucose, we selected ten GK rats as subjects. GK rats are a commonly used model of spontaneous nonobese type 2 diabetes with mildly elevated fasting blood glucose, elevated blood glucose after eating, and stable glucose-stimulated insulin secretion disorders and glucose intolerance (Figure 1A). In addition, it has similar changes to human microvascular complications of type 2 diabetes. Its phenotypes are approaching stability when closing to adulthood. The results demonstrated that postprandial blood glucose in the BD3526 group showed a significant decrease in the fourth and fifth week $\left({ }^{*} P\right.$-value $<0.05$, mean \pm SEM) (Figure 1B). The lowest point of postprandial blood glucose in the BD3526 group appeared in the fifth week, and the
A

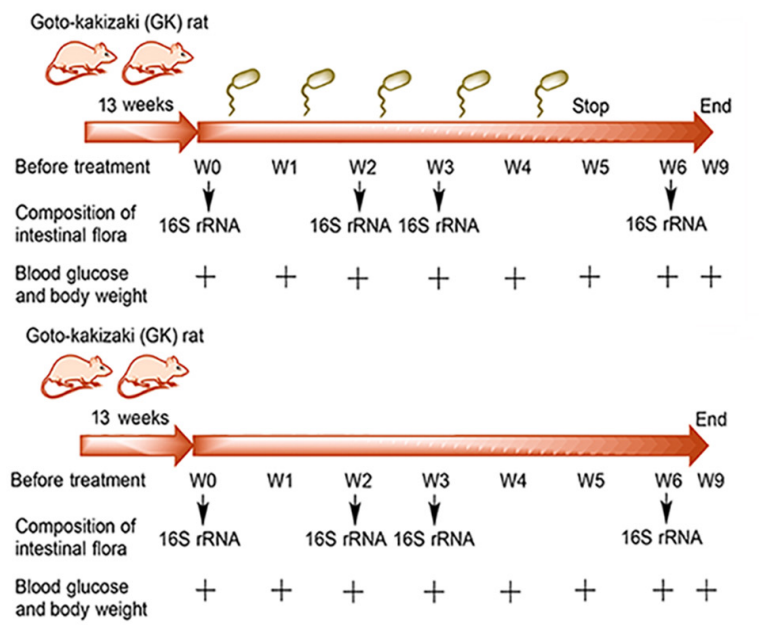

C

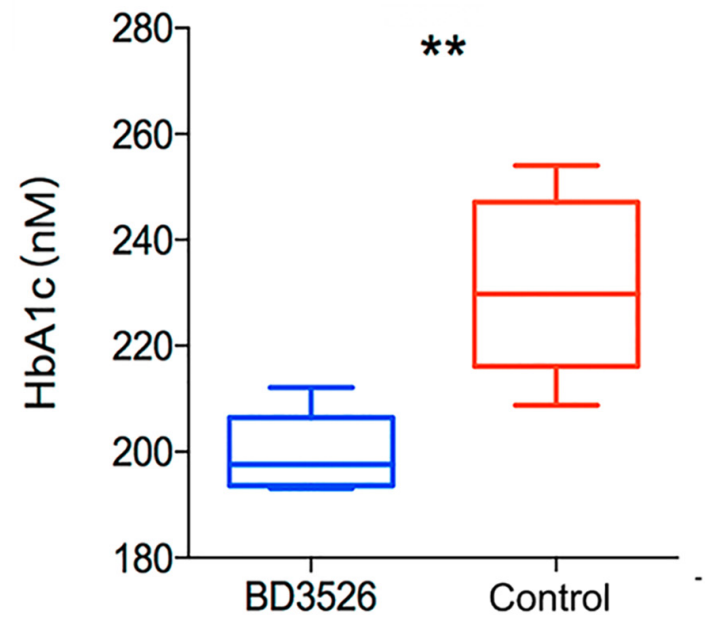

B

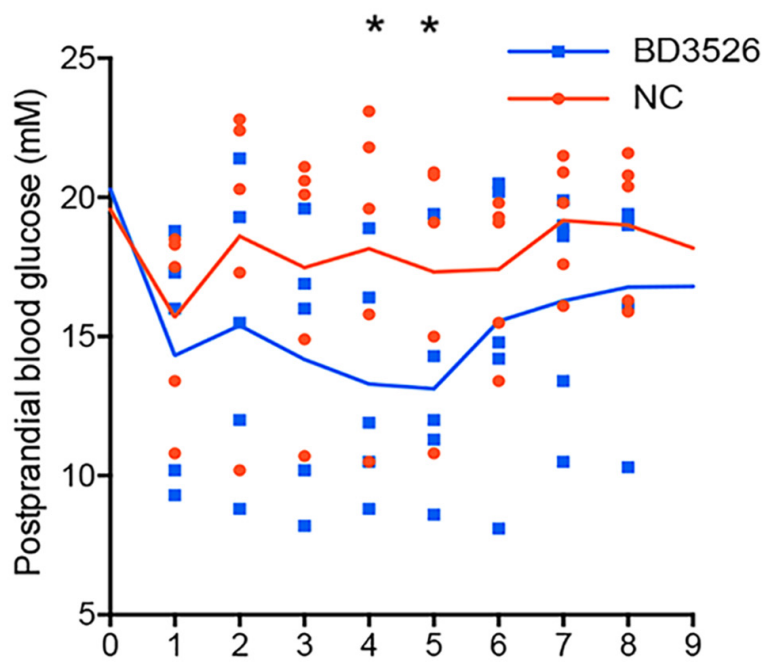

D

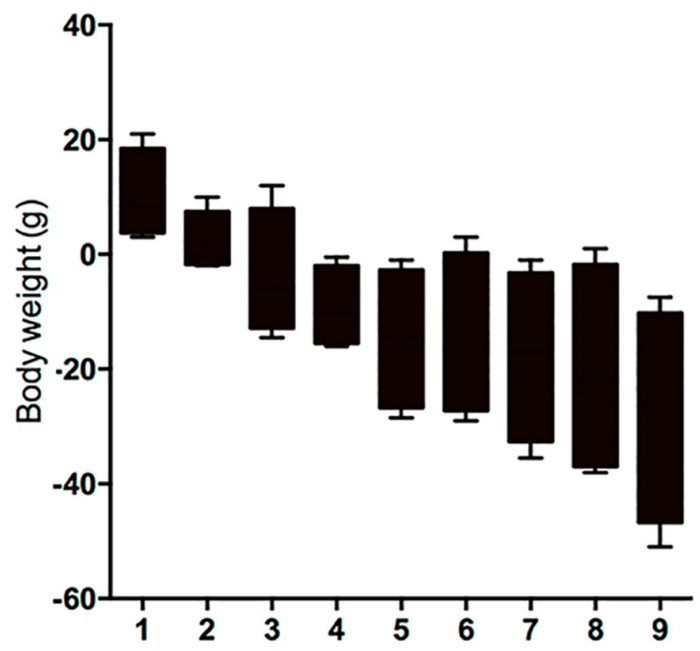

FIGURE 1 | Observation of type 2 diabetes mellitus symptoms. (A) A total of 10, 13-week-old GK rats were used in this work and randomly divided into two groups. The rats in the BD3526 group were gavaged daily with $2 \mathrm{~mL}$ lyophilized powder of the BD3526 strain fermentation products (50 $\mathrm{mg} / \mathrm{mL})$, whereas the GK rats in the control group were gavaged daily with $2 \mathrm{~mL}$ physiological saline. All of the animals were permitted free access to normal chewing bars and water. At intervals of 0,2 , 3 , and 6 weeks, the changes of diabetes indexes in these rats were observed. At the sixth week, the gavage of either BD3526 strain fermentation products or physiological saline was interrupted and the rats were restored to normal diets. After the restoration, the animals were anesthetized and killed, and the mucus in the intestine and colon was scratched. It is desirable to observe changes in the phenotype after stopping the ingestion of the BD3526 strain fermentation products. (B) Postprandial blood glucose measurements were performed in the BD3526 group and the control group. The $x$-axis represents the time of the week. The $y$-axis represents the postprandial blood glucose concentration ( $\mathrm{mM} / \mathrm{L})$. The asterisk represents a significant difference in blood glucose concentration between the BD3526 group and the control group ( ${ }^{*} P$-value $<0.05$, mean \pm SEM). (C) The glycated hemoglobin (HbA1c) test confirmed that the blood glucose concentration in the BD3526 group was significantly lower than that in the control group (** $P$-value $<0.01$, mean \pm SEM). (D) Body weight indicators were used to assess weekly body weight changes in the BD3526 group and control group models (mean \pm SEM). 
blood glucose concentration was $13.12 \mathrm{mM} / \mathrm{L}$. Correspondingly, the postprandial blood glucose concentration of the control group of GK rats was $17.32 \mathrm{mM} / \mathrm{L}$ in the fifth week. In addition, the glycosylated hemoglobin index of the BD3526 group was also significantly lower than that of the control group $\left({ }^{*} P\right.$-value $<0.01$, mean $\left.\pm \mathrm{SEM}\right)$ (Figure $\left.1 \mathrm{C}\right)$. The average concentration of glycated hemoglobin in the BD3526 group was $199.58 \mathrm{nM} / \mathrm{L}$, whereas the value in the control group was $231.25 \mathrm{nM} / \mathrm{L}$. This finding suggests that the diabetic symptoms of the BD3526 group were significantly alleviated. Furthermore, in the body mass index test, we subtracted the body weight of the control group rats from that of the BD3526 group and observed that the weight gain rate of GK rats in the $\mathrm{BD} 3526$ group was significantly lower than that in the control group (Figure 1D). These results indicate that the BD3526 strain fermentation products had the ability to reduce diabetes-related indicators in GK rats of T2DM.

\section{The Diversity of Gut Microbes in GK Rats Fed With the Fermentation Products of the BD3526 Strain Increased}

In addition to weekly testing of physiological and biochemical indicators of GK rats in the BD3526 group and the control group, fecal samples were also collected at weeks $0,2,3$, and 6 for $16 \mathrm{~S}$ rRNA sequencing. It is hoped that the effect on the gut microbes of GK rats can be observed. Among them, week zero and the sixth week were the standards of the initial and washout value of the GK rat fecal microbiota in the BD3526 group, respectively, and the second week and the third week represented the microbiota affected by the intake of the BD3526 fermentation products.

The microbiota in the fecal samples of either the BD3526 group or the control were assayed by 16S rRNA sequencing performed on the Illumina Miseq platform. After sequencing, the biodiversity of the gut microbes of the two groups of GK rats were analyzed. At weeks 2 and 3, Pan/Core analysis showed that in the BD3526 group, the total number of OTUs was significantly higher than that in the control group (Figure 2A). Correspondingly, the number of OTUs shared in both the control group and the $\mathrm{BD} 3526$ group showed a significant decrease (Figure 2B). The Shannon curve also showed that the number of OTUs in the BD3526 group and that in the control group were saturated with the increase in sequencing reads, and the number of OTUs in the BD3526 group was greater than that in the control group (Figure 2D). These results indicate that the gut microbiota diversity of GK rats in the BD3526 group was significantly higher than that in control GK rats. This result was also confirmed by alpha diversity verification $\left({ }^{*} P<0.05\right.$, mean \pm SEM) (Figure 2C).

To perform a difference analysis within and between groups, a PLS-DA (Partial Least Squares Discriminant Analysis) algorithm was chosen to compare the two groups of GK for week 0 , week 2, week 3, and week 6. As shown in Figure 2E, no significant difference between the BD3526 group and the control group was observed. Instead, with the administration of the BD3526 fermentation products, the gut microbiota was diversified, and at the third week, the difference between the BD3526 group and the control group was the most significant. Nevertheless, at the 6th week and after termination of administration of the BD3526 fermentation products for 1 week, the difference in the gut microbiota between the two groups was partially restored.

\section{Akkermansia Is Greatly Enriched in the Gut of GK Rats Fed BD3526 Fermentation Products}

The 16S rRNA sequencing is mainly concerned with differences in gut microbiota composition between groups. Therefore, we used the metagenomeseq algorithm to analyze the differences in gut microbiota composition between BD3526 GK rats and control GK rats at weeks 2 and 3. Based on the statistics of the counts of each group of sequencing samples, we found that a total of 23 genera changed in the BD3526 group $(P<0.05)$. Among them, Akkermansia, Ruminococcaceae_NK4A214, Ruminclostridum_1 and No rank_Peptococcaceae were elevated in the BD3526 group (Figure 3A and Supplementary Table S1). A statistical analysis of the FDR-corrected $P$-values of these 23 genera revealed that Akkermansia demonstrated the most significant difference among all of the changed genera $[-10 \operatorname{Lg}(P$-value $)=2.848796]$ (Figure 3B). In the BD3526 group, Akkermansia, Ruminococcaceae_NK4A214, Ruminiclostridium_1 and Lachnospiraceae_ND3007 were enriched, while the four genera were not detected in the other group. Instead, Alkaliphilus, Sulfurimonas, Amphritea, Photobacterium, Arenibacter, Anaerolineaceae, Flavobacteriaceae, Sulfurovum, Colwellia, Clostridium sensu, Magnetovibrio, Thiotrichaceae, and Rhodobacteraceae disappeared in the BD3526 group. To be precise, after correcting Akkermansia to species, we found that it corresponds to A. muciniphila.

However, the metagenomeseq algorithm itself has certain limitations. To eliminate the ostensible impact of gut microbiota diversity caused by the genus due to its low abundance or low differential fold and efficiently identify the most important OTUs for the BD3526 group, we also constructed a model of random forest distribution (Figure 3C). In this model, the gut microbiota in the BD3526 group and the control group were enriched in two different sites. We found that Akkermansia had the most important position in this model based on the genus abundance of random forest distribution (Figure 3D and Supplementary Table S2). This suggests that Akkermansia may be the biggest influencing factor for the difference between the BD3526 group and the control group. In addition to Akkermansia, No rank_Lachnospiraceae, Prevotella_9, Ruminiclostridium, Lachnospiraceae_UCG_001 and Candidatus_Saccharimonas were also among the top five genera. Among them, Akkermansia and Prevotella_9 were also the two genera screened by the metagenomeseq algorithm that showed a significant difference between the two rat groups.

In the random forest distribution analysis, we also used the top 6 genera of the mean reduction accuracy (Akkermansia, No rank_Lachnospiraceae, Prevotella_9, Ruminiclostridium, Lachnospiraceae_UCG_001 and Candidatus_Saccharimonas) as models to perform the receiver operating characteristic curve 


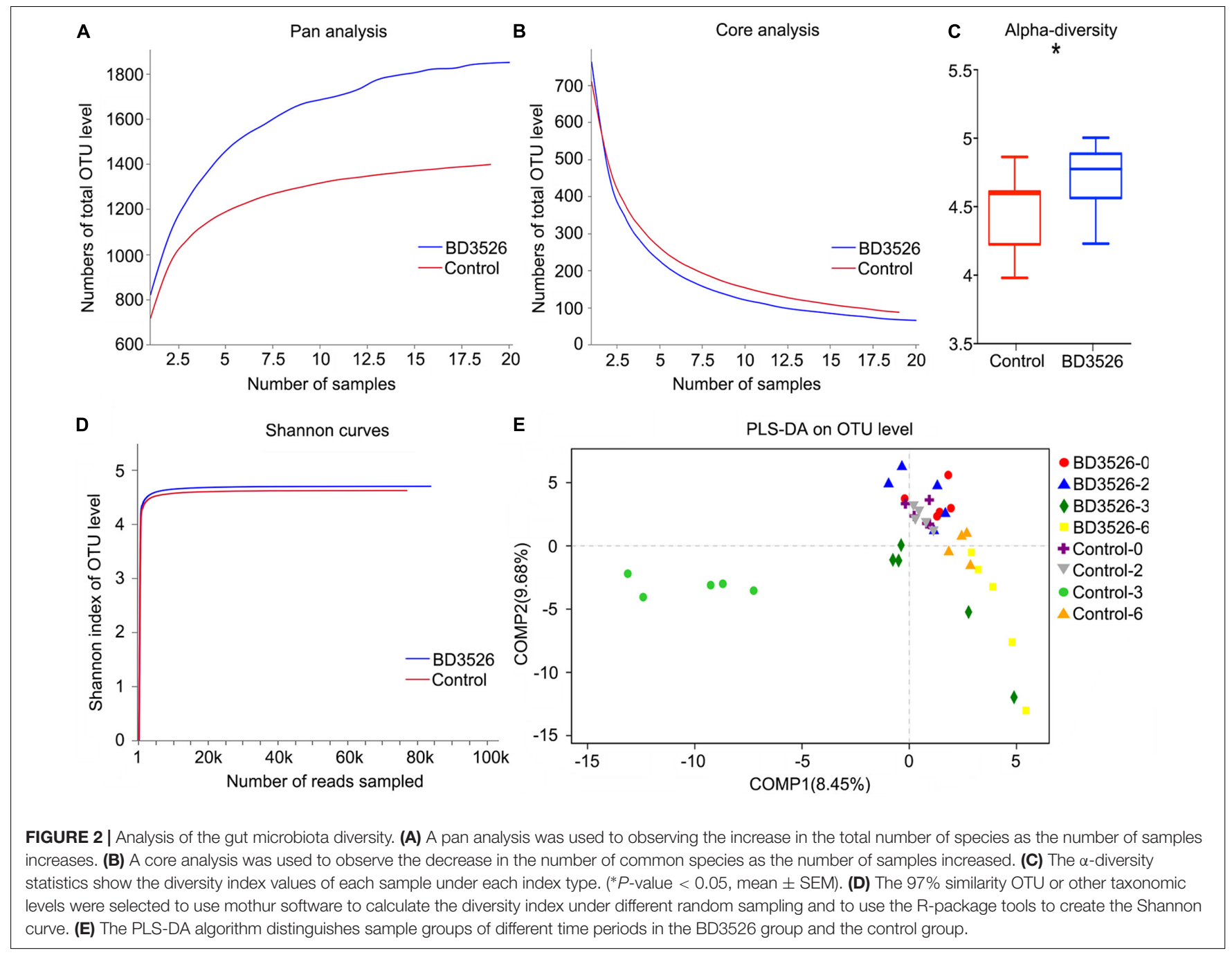

analysis (ROC curve) (Supplementary Figure S1). The results show that in the model containing only these six genera, the Receiver Operating Characteristic Curve (ROC) reached 0.81. Correspondingly, in the model containing all genera, the ROC is 0.48 . When the six genera were removed from the model of all genera, the ROC was 0.51 . This illustrated that our model was accurate to assess the difference between the two groups.

Interestingly, among the six genera, Akkermansia displayed the most significant difference in 16S rRNA sequencing, which suggests that Akkermansia may play an important role in ameliorating the symptoms of the GK rats fed BD3526 fermentation products. Although short-chain fatty acids (SCFAs) had been reported as key effectors in T2DM by other researchers (Zhao et al., 2018), no enrichment of SCFAs-producing microbiota was observed in the BD3526 group.

For further confirm the direct relationship between fermentation products and Akkermansia, we re-cultured the fecal samples in vitro which treated with 5\% fermentation products or 5\% skim milk. In this in vitro experiment, we focused Akkermansia was significantly enriched after treated with fermentation products for 1 day (Supplementary Figure S2).
This phenomenon was consistent to the conclusions we claimed before that Akkermansia is greatly enriched in the gut of GK rats fed BD3526 fermentation products.

\section{The BD3526 Strain Fermentation Products Shifted the Interactions of the Gut Microbes}

In the gut, microbe interactions between different genera and between different species often occurred. Therefore, to further evaluated the effect of the BD3526 strain fermentation products on the gut microbiota of GK rats, we used Networkx software for interaction network analysis. The interaction between microorganisms of different genera in the same sample and species correlation between different samples were evaluated. In the species interaction network of the BD3526 group and the control group, we found that the dominant species belong to Firmicutes. At the phylum level, there was no significant difference between the two groups (Figures 4A,B). However, when we constructed the node centrality diagram at the genus level, we found that the 


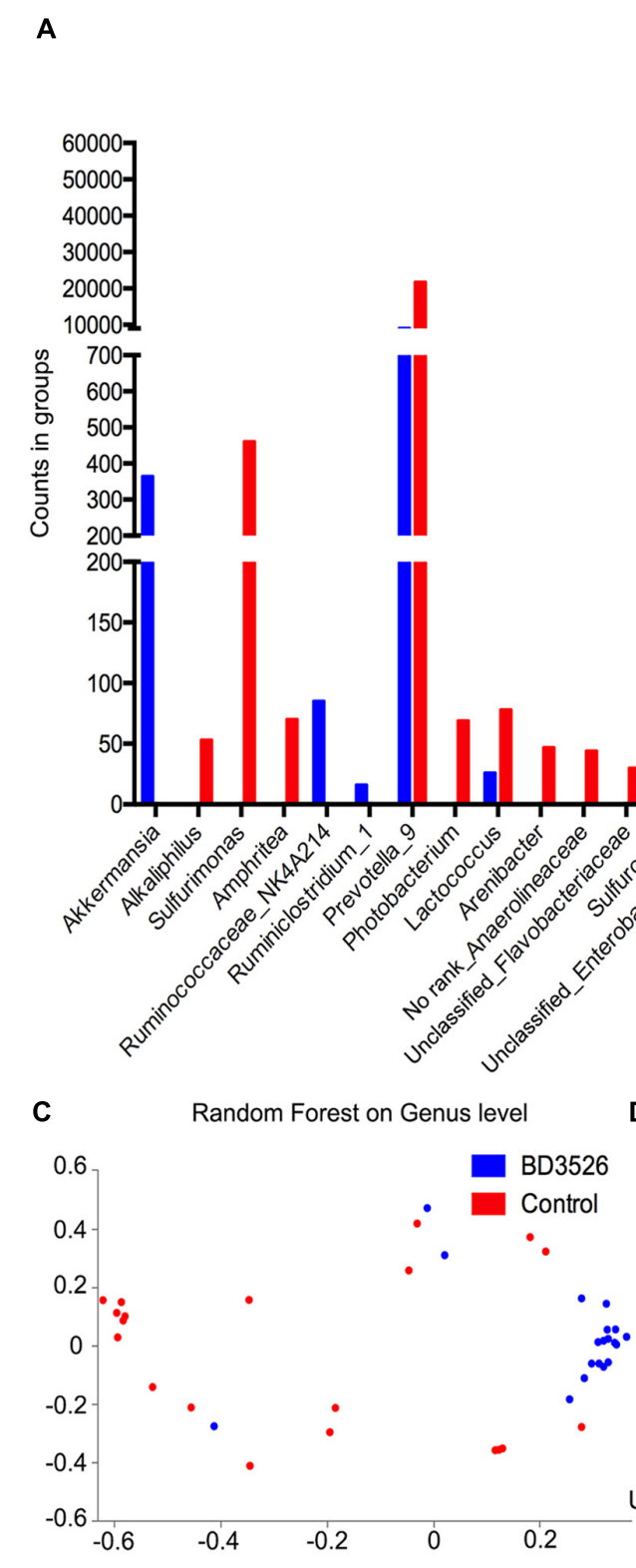

B

BD3526 Control

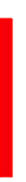
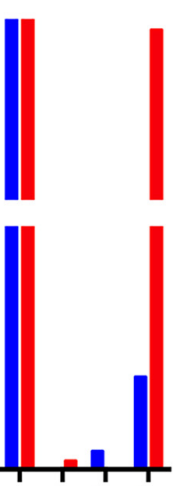

B $\quad-\operatorname{Lg}($ P-value $)$

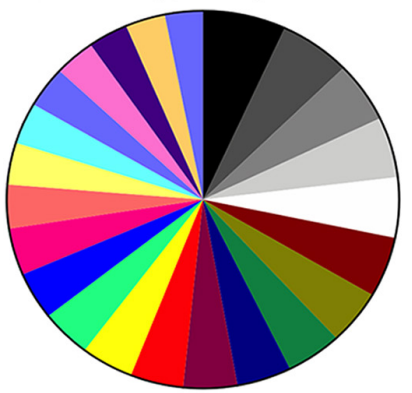

2.848796 Akkermansia 2.446390 Alkaliphilus

2.167367 Sulfurimonas

2.159028 Amphritea

$\square$ 2.155024 Ruminococcaceae_NK4A214

2.154731 Ruminiclostridium_1

$\square 1.951983$ Prevotella_9

$\square 1.908951$ Photobacterium

1.867034 Lactococcus

- 1.853365 Arenibacter

$\square \quad 1.819317$ No rank Anaerolineaceae

$\square 1.801450$ Unclassified_Flavobacteriaceae

$\square \quad 1.801450$ Unclassified
$\square \quad 1.724162$ Sulfurovum

- 1.700144 Unclassified_Enterobacteriaceae

$\square 1.673936$ Colwellia

$\square 1.561036$ Clostridium_sensu

$\square 1.561036$ Clostridium_sensu

$\square$ 1.477055 Magnetovibrio

$\square 1.474709$ Thiotrichaceae

1.397854 Lactobacillus

1.368394 Unclassified_Rhodobacteraceae

$\square$ 1.366817 Lachnospiraceae_ND3007

$\square 1.302210$ Coprococcus 2

D

No rank_Lachnospiraceae

Prevotella_9

Ruminiclostridium

Lachnospiraceae_UCG_001

- Candidatus_Saccharimonas

Lachnospiraceae_UCG_010

Arenibacter

Anaerotruncus

Unclassified_Flavobacteriaceae

0

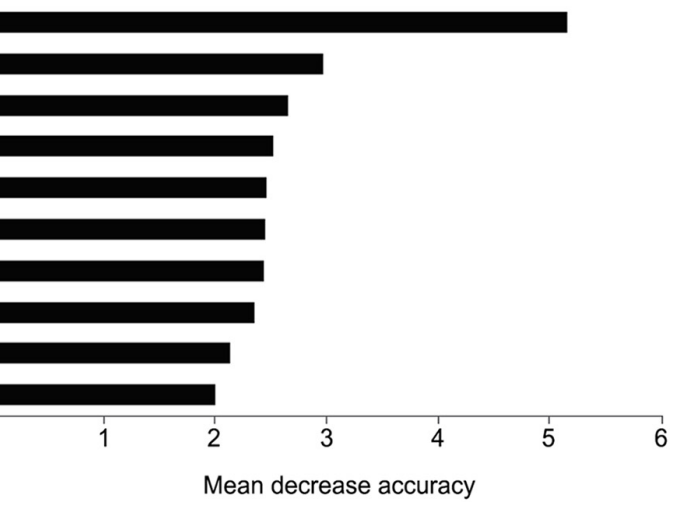

FIGURE 3 | Screening of the different genera. (A) A metagenomeseq method was used to screen different strains of the BD3526 group and control group. The blue columns represent the enrichment of different strains in the BD3526 group. The red columns represent the enrichment of different strains in the control group.

(B) P-value distribution of differential strains screened by metagenomeseq method. (C) Two-dimensional scatter plot prediction of BD3526 and control samples by random forest distribution. (D) Rapid selection of species categories that are most important for sample classification through random forest distribution. The highest mean decrease accuracy of the genus represented the greatest impact on the BD3526 and control components.

interaction network of the BD3526 group and the control group were significantly different. In the node centrality diagram, the number on the $x$-axis represents a different node. Each node corresponds to the interaction of the individual genus to the other genera. When a peak simultaneously occurred on the three curves of the degree centrality, closeness centrality and between centrality, it was considered that the corresponding genus of the node might be of great significance to the entire network. According to this principle,
9 genera were identified in the BD3526 group including No rank_Ruminococcaceae, Butyrivibrio, Prevotellaceae_NK3B31, Parasutterella and Lactobacillus, Ruminococcaceae_UCG014, Ruminococcaceae_UCG-013, Treponema_2 and Ruminiclostridium. In the control group, only five genera were identified including Unclassified_Veillonellaceae, Sulfurovum, Oscillibacter, No rank_Gastranaerophilales, and [Ruminococcus]_gauvreauii_group (Figures 4C,D). There were no associations between the 9 genera of the BD3526 group 
A

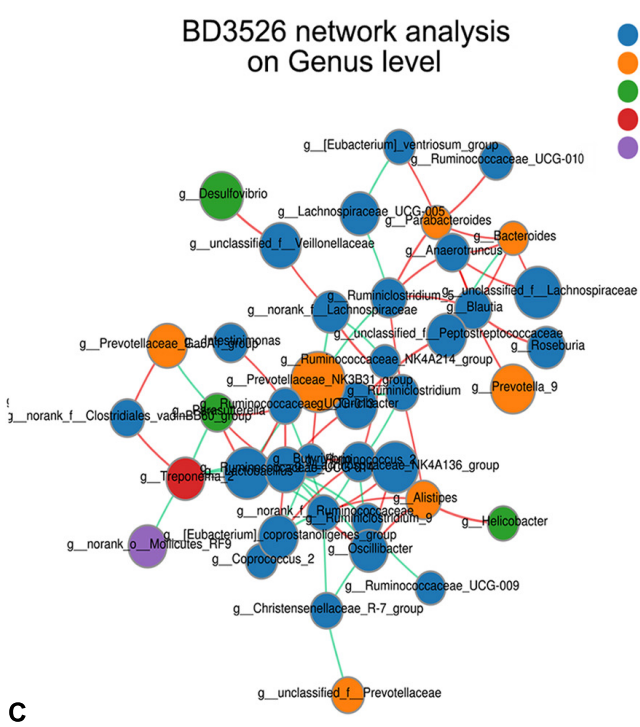

Node centrality analysis on Genus level
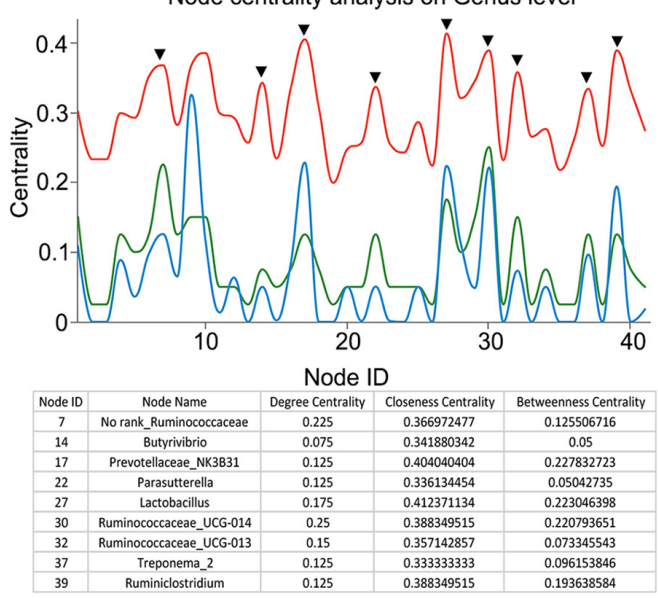

B

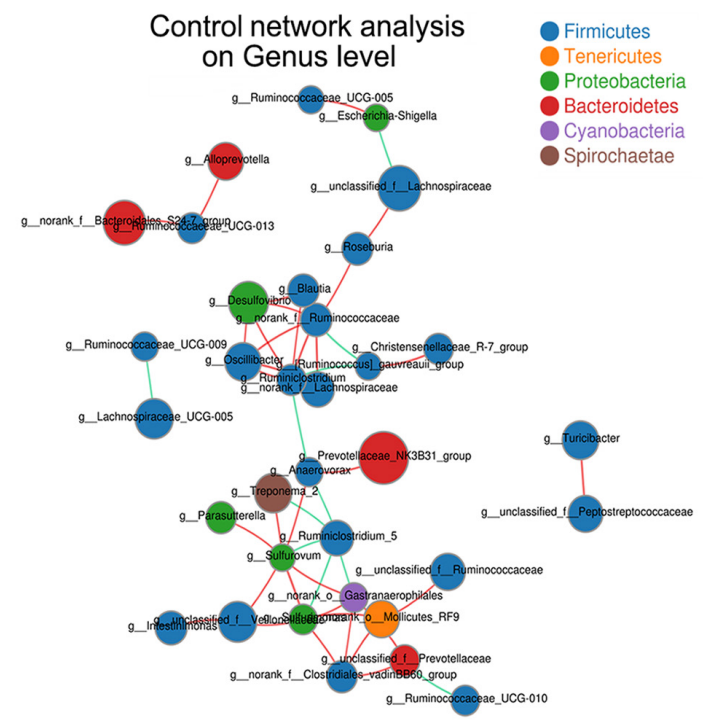

D
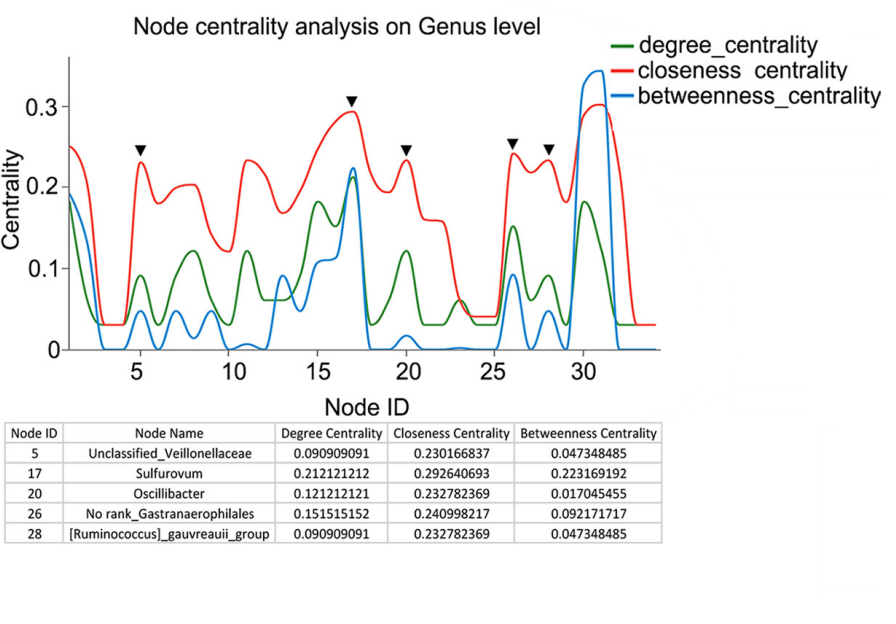

FIGURE 4 | Interaction network of gut microbiota. (A) The dominant microbiota interaction network in the BD3526 group. (B) The dominant microbiota interaction network in the control group. (C) The key strain nodes in the BD3526 group were analyzed for the core genus. The green curve represents the degree centrality. The red curve represents the closeness centrality and the blue curve represents the betweenness centrality. The nodes whose three curves reach the peak at the same time may be the genus that plays an important role in the interaction network. The detailed genus name and parameter information are listed in the following table. The black triangles represent the important genus in this network. (D) The key strain nodes in the BD3526 group were analyzed for the core genus. The green curve represents the degree centrality. The red curve represents the closeness centrality and the blue curve represents the betweenness centrality. The nodes whose three curves reach the peak at the same time may be the genus that plays an important role in the interaction network. Detailed genus name and parameter information are listed in the following table. The black triangles represent the important genus in this network.

and the 5 genera of the control group. This result suggests that the fermentation products of the strain BD3526 may exert an important impact on the gut microbiota interaction network of GK rats. This may be another important factor to improve the symptoms of T2DM in the $\mathrm{BD} 3526$ group.

At the same time, the species correlation revealed that the rats in the BD3526 strain group shared a portion of the species with those of the control group. However, the points corresponding to the BD3526 group alone were significantly higher than those of the control group alone (Supplementary Figure S3), which indicated that the species in the gut microbiota of GK rats in the BD3526 group demonstrated more specificity.

\section{The BD3526 Strain Fermentation Products Decreased the Genera in the Gut Microbiota That Were Closely Related to Diseases in GK Rats}

To assess the effect of different microbes on the stability of the gut microbiota in the BD3526 group, we obtained $16 \mathrm{~S}$ rRNA sequencing data through the corresponding Greengene ID of each OTU. The functional composition profiles were predicted with PICRUSt. The results show that in the BD3526 group, the most significant changes in KEGG (level 2) categories were focused on five pathways including immune 
system diseases $[-10 \quad \mathrm{Lg}(P$-value $)=17.798790]$, cancer $[-10 \mathrm{Lg}(P$-value $)=16.241620]$, cell growth and death $[-10 \mathrm{Lg}(P$-value $)=15.919890]$, translation $[-10 \mathrm{Lg}(P-$ value $)=16.241620]$ and infectious diseases $[-10 \mathrm{Lg}(P$ value $)=15.785920$ ] (Figure 5). These five KEGG pathways were all associated with diseases including immune disease, cancer and infectious diseases. This suggests that the intake of the BD3526 strain fermentation products may have a profound effect on the development of diseases and maintain the balance of the gut microbiota of GK rats.

\section{The BD3526 Strain Fermentation Products Lowered the Intestinal Mucosal Inflammation Response in GK Rats}

The causes of T2DM are often complicated. Studies have reported that the onset of T2DM was correlated with a chronically lowgrade inflammatory response (Donath et al., 2003; Ehses et al., 2009; Masters et al., 2010; Westwellroper et al., 2011; Lerner et al., 2012; Oslowski et al., 2012; Jourdan et al., 2013; WestwellRoper et al., 2014). The inflammatory response plays an important role in the occurrence and development of diabetes. Another study reported that $A$. muciniphila can increase intestinal mucus thickness and reduce the inflammation response ( $\mathrm{Wu}$ et al., 2017).

As aforementioned, we found that the BD3526 strain fermentation products significantly increased A. muciniphila in $\mathrm{GK}$ rats and reduced the genera in the gut microbiota closely related to diseases by the KEGG analysis. Therefore,

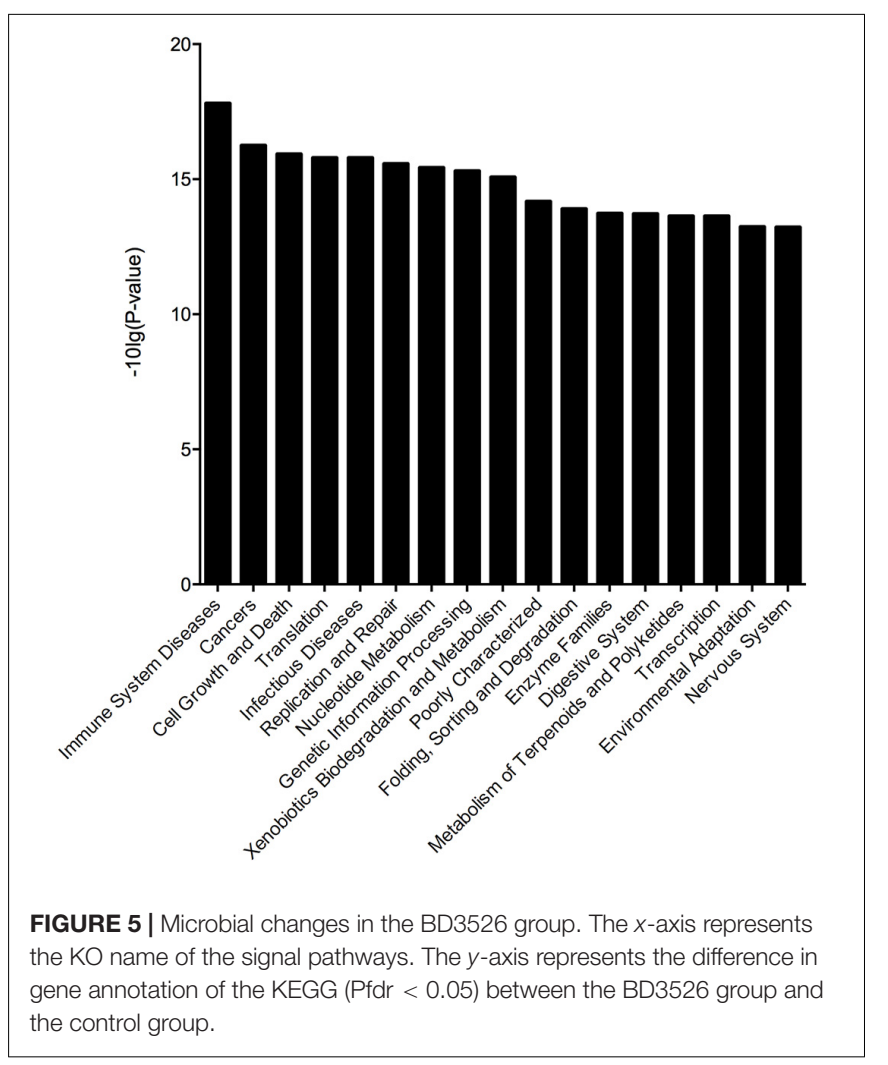

we postulated that the BD3526 strain fermentation products could reduce the inflammatory factors of intestinal mucosal by increasing the A. muciniphila content and ultimately play a role in lowering blood glucose. Assuming this postulation, we extended our work to the inflammatory factors in the intestinal mucosa of the BD3526 group and the control group by using a rat Cytokine Antibody Array Kit (Abcam, ab133992). After a period of 5 weeks of washout, the cytokines expressed in the large intestinal mucus of the rats in the BD3526 group and those in the control group were assayed. The kit can simultaneously detect 34 cytokines in one array. In the large intestinal mucus of the rats in the control group, 32 cytokines, including inflammatory factors (IL-1 $\beta$, IL-6, MCP-1 and TNF- $\alpha$ ) and other oncogenic factors, were expressed at a high level in the GK rats, whereas the Neg and Fas ligand could hardly be detected (Figures 6A,B). Among the 32 cytokines increasingly expressed, an oncogenic factor Agrin (A6 and B6 spots in the array map) was remarkably enriched in GK rats. In the other 31 cytokines, IL-1 $\beta$ (C7 and D7 spots in the array map) is regarded as a cytokine that activates multiple immune cells and promotes insulin resistance (Dinarello, 2009). IFN- $\gamma$ (C5 and D5 spots in the array map) is reported to be a mediator in the regulation of glucose metabolism by $A$. muciniphila (Greer et al., 2016). IL-6 (C11 and D11 spots in the array map) is confirmed to be a promoter of the death of islet $\beta$ cells, which leads to T2DM (Donath, 2013). Correspondingly, the expression of cytokines in the BD3526 group was generally decreased (Figures 6A,B). To further semi-quantify the expression of inflammatory factors, we used ImageJ software to perform gray-scale analysis on the two images. The results showed that the intensity of the positive controls in the upper left and lower right are similar in the two arrays. However, the only expressed gene Agrin in the BD3526 group decreased by approximately $50 \%$ compared with the control group (Figure 6C). This result implied that the BD3526 strain fermentation products could reduce the expression level of most cytokines in the large intestinal mucus, such as IL- $1 \beta$, IFN- $\gamma$ and IL-6, and thus lower the blood glucose in GK rats.

\section{DISCUSSION}

The gut microbiota is widely involved in the development of various diseases, and alteration of the gut microbiota through the intake of some specific microbial supplements has been adopted for the treatment of various diseases. These diseases include autism, Parkinson's disease and cancer (Hsiao et al., 2013; Buffington et al., 2016; Thaiss et al., 2016; Yu et al., 2017; Chung et al., 2018; Dejea et al., 2018; Routy et al., 2018; Tilg et al., 2018). In the study of gut microbiota and diabetes, the diversity and composition of the gut microbiota of patients changed significantly compared with healthy people (Cani et al., 2008; Larsen et al., 2010; Musso et al., 2010; Qin et al., 2012). In several intervention experiments, metformin has been found to increase the content of A. muciniphila and other short-chain fatty acid-producing microorganisms in the intestine of diabetic patients (Shin et al., 2014; de la Cuesta-Zuluaga et al., 2017; Wu et al., 2017; Wang et al., 2018). Metformin also has a profound effect on the interaction of microorganisms within the 
A Control group BD3526 group

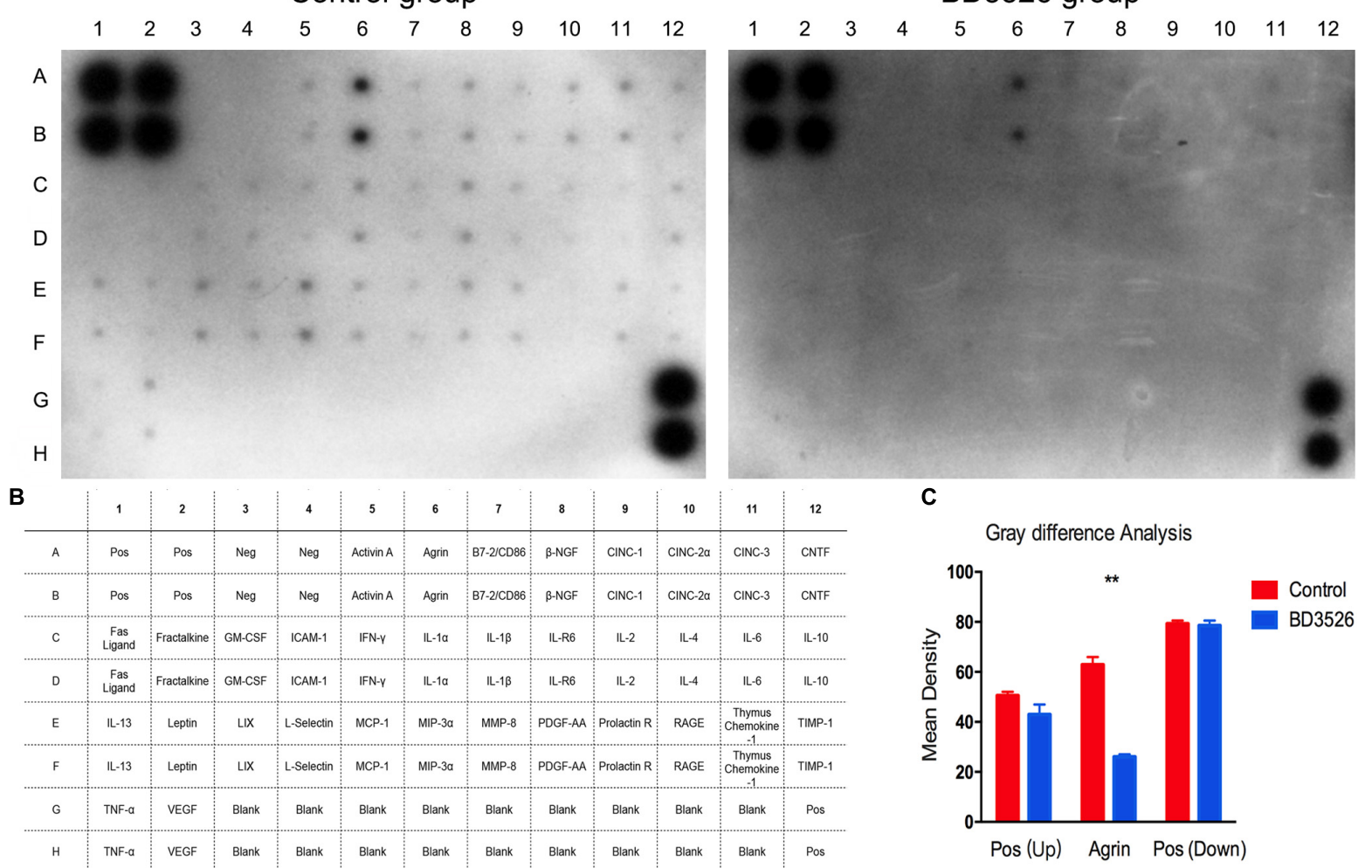

FIGURE 6 | Intestinal mucosal cytokines detection. (A) The cytokines of the control group and the BD3526 group were both detected. (B) In the membrane, all of the spots were listed in the table below. In addition to all 34 cytokines, A1, A2, B1, B2, G12, and H12 were positive controls. G3 to G11 and $\mathrm{H} 3$ to $\mathrm{H} 11$ were all negative controls. (C) Cytokines were semi-quantitatively analyzed using ImageJ software (** $P$-value $<0.01$, mean \pm SEM).

gut microbiota. In this work, when GK rats were administered with the BD3526 strain fermentation products, the content of A. muciniphila in the intestine increased significantly compared with those in the control group. Similarly, A. muciniphila showed an increase after the metformin treatment in T2DM subjects (Shin et al., 2014; de la Cuesta-Zuluaga et al., 2017). This outcome indicates that metformin and the BD3526 strain fermentation products might behave similarly in alleviating the symptoms of diabetes. However, administration of metformin not only increase the content of $A$. muciniphila but also the content of other SCFAs-producing microorganisms in the intestine, e.g., Butyrivibrio, Bifidobacterium bifidum, and Megasphaera (de la Cuesta-Zuluaga et al., 2017), which provide a rational explanation for the higher levels of SCFAs being detected in the stool of clinical patients taking metformin (Wu et al., 2017). A large amount of dietary fiber has also been demonstrated to promote the growth of some probiotics in the intestine, stimulate the production of SCFAs, and alleviate the symptoms of diabetes (Zhao et al., 2018). Nevertheless, GK rats fed the BD3526 strain fermentation products did not exhibit a similar phenomenon, i.e., SCFAs-producing microorganisms in the BD3526 group were not wholly increased. Therefore, it could be postulated that both A. muciniphila and SCFAs-producing microorganisms are important in the regulation of blood glucose and behaved differently.
Akkermansia muciniphila is an anaerobic bacterium attached to the intestinal mucosa (Derrien et al., 2004, 2008; Collado et al., 2007; van Passel et al., 2011), and a lower level of A. muciniphila has been reported in obesity and T2DM subjects compared with normal ones. In addition, A. muciniphila can also increase the patient's response to drugs by increasing the recruitment of $\mathrm{CCR}^{+} \mathrm{CXCR}^{+}{ }^{+} \mathrm{CD}^{+}{ }^{+} \mathrm{T}$ lymphocytes during the immunotherapy of tumor patients with PD-1/PDL1 and increase the progression-free survival (PFS) of patients after treatment (Routy et al., 2018). Although the molecular mechanism by which $A$. muciniphila ameliorates diabetes/obesity is not fully understood, it is widely believed that this bacterium can play a positive role in health promotion by increasing the integrity of the intestinal mucosa (Derrien et al., 2004, 2008; Collado et al., 2007).

In T2DM, although the direct cause of the syndrome is insufficient for insulin secreted by pancreatic $\beta$-cells, the intrinsic cause is the inflammatory response (Cuman et al., 2001; Naguib et al., 2004). The chronically low grade of inflammatory responses not only causes pancreatic $\beta$-cells to be attacked but also reduces the liver and muscle sensitivity to insulin, which results in insulin resistance (Cerasi, 1995; Kahn, 1998; Bonner-Weir, 2000; Kahn et al., 2006). Even less optimistic is that the inflammatory response not only causes diabetes but also shows correlation between T2DM, insulin resistance, cardiovascular disease and 
obesity (Donath, 2013). Therefore, reducing inflammation has been an important strategy for diabetes treatment. In our work, we found that the BD3526 strain fermentation products could lower the expression of intestinal mucosal inflammatory factors in GK rats and the level of $\mathrm{HaB1c}$ and blood glucose.

In this work, we used the BD3526 strain isolated from Tibetan yak milk to ferment skim milk (Hang et al., 2016). Although our understanding of this strain is not comprehensive, it has already demonstrated some potential in health promotion, e.g., secreting antimicrobial agents and levan, which is a recognized dietary fiber (Xu et al., 2016). Since these fermentation products could alleviate the symptoms of T2DM and selectively stimulate the propagation of $A$. muciniphila in the intestine, we believe that the biological function of $P$. bovis sp. nov. BD3526 might not be restricted to improving diabetes. As only the metabolites of $P$. bovis sp. nov. BD3526 in skim milk are employed in this work, the effect of the bacterium itself on the intestinal tract is still unknown and worthy of further investigation.

\section{AUTHOR CONTRIBUTIONS}

$\mathrm{ZQ}, \mathrm{JH}, \mathrm{ZW}$, and $\mathrm{ZL}$ conceived and designed the experiments. $\mathrm{JH}, \mathrm{HF}, \mathrm{MY}, \mathrm{CG}, \mathrm{JW}$, and CY performed the experiments.

\section{REFERENCES}

André-Schmutz, I., Hindelang, C., Benoist, C., and Mathis, D. (2010). Cellular and molecular changes accompanying the progression from insulitis to diabetes. Eur. J. Immunol. 29, 245-255. doi: 10.1002/(SICI)1521-4141(199901) 29:01<245::AID-IMMU245>3.0.CO;2-O

Bonner-Weir, S. (2000). Islet growth and development in the adult. J. Mol. Endocrinol. 24, 297-302. doi: 10.1677/jme.0.0240297

Buffington, S., Diprisco, G. V., ROChtung, T., Ajami, N., Petrosino, J., and CostaMattioli, M. (2016). Microbial reconstitution reverses maternal diet-induced social and synaptic deficits in offspring. Cell 165, 1762-1775. doi: 10.1016/j.cell. 2016.06.001

Cani, P. D., Bibiloni, R., Knauf, C., Waget, A., Neyrinck, A. M., Delzenne, N. M., et al. (2008). Changes in gut microbiota control metabolic endotoxemiainduced inflammation in high-fat diet-induced obesity and diabetes in mice. Diabetes 57, 1470-1481. doi: 10.2337/db07-1403

Cerasi, E. (1995). Insulin deficiency and insulin resistance in the pathogenesis of NIDDM: is a divorce possible? Diabetologia 38, 992-997. doi: 10.1007/ BF00400591

Chung, L., Orberg, E. T., Geis, A. L., Chan, J. L., Fu, K., Destefano, C. S., et al. (2018). Bacteroides fragilis toxin coordinates a pro-carcinogenic inflammatory cascade via targeting of colonic epithelial cells. Cell Host Microbe 23, 203.e5214.e5. doi: 10.1016/j.chom.2018.01.007

Collado, M. C., Derrien, M., Isolauri, E., de Vos, W. M., and Salminen, S. (2007). Intestinal integrity and Akkermansia muciniphila, a mucin-degrading member of the intestinal microbiota present in infants, adults, and the elderly. Appl. Environ. Microbiol. 73, 7767-7770. doi: 10.1128/AEM.01477-07

Coughlin, S. S., Calle, E. E., Teras, L. R., Petrelli, J., and Thun, M. J. (2004). Diabetes mellitus as a predictor of cancer mortality in a large cohort of US adults. Am. J. Epidemiol. 159, 1160-1167. doi: 10.1093/aje/kwh161

Cuman, R. K., Bersani-Amado, C. A., and Fortes, Z. B. (2001). Influence of type 2 diabetes on the inflammatory response in rats. Inflamm. Res. 50, 460-465. doi: 10.1007/PL00000271

de la Cuesta-Zuluaga, J., Mueller, N. T., Corrales-Agudelo, V., Velãsquez-Mejiã, E. P., Carmona, J. A., Abad, J. M., et al. (2017). Metformin is associated with higher relative abundance of mucin-degrading Akkermansia muciniphila and several short-chain fatty acid-producing microbiota in the gut. Diabetes Care 40, 54-62. doi: $10.2337 / \mathrm{dc} 16-1324$
ZQ, HZ, CG, and CY analyzed the data. ZQ and JH wrote the manuscript. All the authors read and approved the final manuscript.

\section{FUNDING}

This project was sponsored by Shanghai Engineering Center of Dairy Biotechnology (16DZ2280600) and the Shanghai RisingStar Program (18QB1400100).

\section{ACKNOWLEDGMENTS}

We would like to thank Huifang An from Sinotech Genome Technology Co., Ltd., for the 16S rRNA sequencing data analysis for this work.

\section{SUPPLEMENTARY MATERIAL}

The Supplementary Material for this article can be found online at: https://www.frontiersin.org/articles/10.3389/fmicb. 2018.03292/full\#supplementary-material

Dejea, C. M., Fathi, P., Craig, J. M., Boleij, A., Taddese, R., Geis, A. L., et al. (2018). Patients with familial adenomatous polyposis harbor colonic biofilms containing tumorigenic bacteria. Science 359, 592-597. doi: 10.1126/science. aah3648

Derrien, M., Collado, M. C., Benamor, K., Salminen, S., and de Vos, W. M. (2008). The Mucin degrader Akkermansia muciniphila is an abundant resident of the human intestinal tract. Appl. Environ. Microbiol. 74, 1646-1648. doi: 10.1128/ AEM.01226-07

Derrien, M., Vaughan, E. E., Plugge, C. M., and de Vos, W. M. (2004). Akkermansia muciniphila gen. nov., sp. nov., a human intestinal mucin-degrading bacterium. Int. J. Syst. Evol. Microbiol. 54, 1469-1476. doi: 10.1099/ijs.0. 02873-0

Dinarello, C. A. (2009). Immunological and inflammatory functions of the interleukin-1 family. Annu. Rev. Immunol. 27, 519-550. doi: 10.1146/annurev. immunol.021908.132612

Donath, M. Y. (2013). Targeting inflammation in the treatment of type 2 diabetes. Diabetes Obes. Metab. 15, 193-196. doi: 10.1111/dom.12172

Donath, M. Y., Størling, J., Maedler, K., and Mandruppoulsen, T. (2003). Inflammatory mediators and islet beta-cell failure: a link between type 1 and type 2 diabetes. J. Mol. Med. 81, 455-470. doi: 10.1007/s00109-003-0450-y

Ehses, J. A., Ellingsgaard, H., Bönjschnetzler, M., and Donath, M. Y. (2009). Pancreatic islet inflammation in type 2 diabetes: from alpha and beta cell compensation to dysfunction. Arch. Int. Physiol. 115, 240-247. doi: 10.1080/ 13813450903025879

Everard, A., Belzer, C., Geurts, L., Ouwerkerk, J. P., Druart, C., Bindels, L. B., et al. (2013). Cross-talk between Akkermansia muciniphila and intestinal epithelium controls diet-induced obesity. Proc. Natl. Acad. Sci. U.S.A. 110, 9066-9071. doi: $10.1073 /$ pnas. 1219451110

Forslund, K., Hildebrand, F., Nielsen, T., Falony, G., Le, C. E., Sunagawa, S., et al. (2017). Disentangling type 2 diabetes and metformin treatment signatures in the human gut microbiota. Nature 545:116. doi: 10.1038/nature22318

Greer, R. L., Dong, X., Moraes, A. C. F., Zielke, R. A., Fernandes, G. R., Peremyslova, E., et al. (2016). Akkermansia muciniphila mediates negative effects of IFN $\gamma$ on glucose metabolism. Nat. Commun. 7:13329. doi: 10.1038/ ncomms 13329

Hang, F., Liu, P., Wang, Q., Han, J., Wu, Z., Gao, C., et al. (2016). High milkclotting activity expressed by the newly isolated Paenibacillus spp. Strain BD3526. Molecules 21:73. doi: 10.3390/molecules21010073 
Hsiao, E. Y., McBride, S. W., Hsien, S., Sharon, G., Hyde, E. R., McCue, T., et al. (2013). Microbiota modulate behavioral and physiological abnormalities associated with neurodevelopmental disorders. Cell 155, 1451-1463. doi: 10. 1016/j.cell.2013.11.024

Jourdan, T., Godlewski, G., Cinar, R., Bertola, A., Szanda, G., Liu, J., et al. (2013). Activation of the Nlrp3 inflammasome in infiltrating macrophages by endocannabinoids mediates beta cell loss in type 2 diabetes. Nat. Med. 19, 1132-1140. doi: 10.1038/nm.3265

Kahn, B. B. (1998). Type 2 diabetes: when insulin secretion fails to compensate for insulin resistance. Cell 92, 593-596. doi: 10.1016/S0092-8674(00)81125-3

Kahn, S. E., Hull, R. L., and Utzschneider, K. M. (2006). Mechanisms linking obesity to insulin resistance and type 2 diabetes. Nature 444, 840-846. doi: 10.1038 /nature 05482

Karlsson, F. H., Tremaroli, V., Nookaew, I., Bergström, G., Behre, C. J., Fagerberg, B., et al. (2013). Gut metagenome in European women with normal, impaired and diabetic glucose control. Nature 498, 99-103. doi: 10.1038/ nature 12198

Larsen, N., Vogensen, F. K., Fw, V. D. B., Nielsen, D. S., Andreasen, A. S., Pedersen, B. K., et al. (2010). Gut microbiota in human adults with type 2 diabetes differs from non-diabetic adults. PLoS One 5:e0009085. doi: 10.1371/journal.pone. 0009085

Lee, H., and Ko, G. P. (2014). Effect of metformin on metabolic improvement and gut microbiota. Appl. Environ. Microbiol. 80, 5935-5943. doi: 10.1128/AEM. 01357- 14

Lerner, A. G., Upton, J. P., Praveen, P. V., Ghosh, R., Nakagawa, Y., Igbaria, A., et al. (2012). IRE1 $\alpha$ induces thioredoxin-interacting protein to activate the NLRP3 inflammasome and promote programmed cell death during endoplasmic reticulum stress. Cell Metab. 16, 250-264. doi: 10.1016/j.cmet.2012.07.007

Masters, S. L., Dunne, A., Subramanian, S. L., Hull, R. L., Tannahill, G. M., Sharp, F. A., et al. (2010). Activation of the Nlrp3 inflammasome by islet amyloid polypeptide provides a mechanism for enhanced IL- $1 \beta$ in type 2 diabetes. Nat. Immunol. 11, 897-904. doi: 10.1038/ni.1935

Musso, G., Gambino, R., and Cassader, M. (2010). Obesity, diabetes, and gut microbiota. Diabetes Care 33, 2277-2284. doi: 10.2337/dc10-0556

Naguib, G., Al-Mashat, H., Desta, T., and Graves, D. T. (2004). Diabetes prolongs the inflammatory response to a bacterial stimulus through cytokine dysregulation. J. Invest. Dermatol. 123, 87-92. doi: 10.1111/j.0022-202X.2004. 22711.x

Oslowski, C. M., Hara, T., O’Sullivanmurphy, B., Kanekura, K., Lu, S., Hara, M., et al. (2012). Thioredoxin-interacting protein mediates ER stress-induced $\beta$ cell death through initiation of the inflammasome. Cell Metab. 16, 265-273. doi: 10.1016/j.cmet.2012.07.005

Qin, J., Li, Y., Cai, Z., Li, S., Zhu, J., Zhang, F., et al. (2012). A metagenomewide association study of gut microbiota in type 2 diabetes. Nature 490, 55-60. doi: $10.1038 /$ nature 1450

Rotter, V., Nagaev, I., and Smith, U. (2002). Interleukin-6 (IL-6) reduces gene and protein expression of IRS-1 and GLUT4 and is overexpressed in human fat cells from insulin-resistant subjects. Diabetes 51:A303.

Routy, B., Le, C. E., Derosa, L., Cpm, D., Alou, M. T., Daillére, R., et al. (2018). Gut microbiome influences efficacy of PD-1-based immunotherapy against epithelial tumors. Science 359, 91-97. doi: 10.1126/science.aan3706

Shin, N. R., Lee, J. C., Lee, H. Y., Kim, M. S., Whon, T. W., Lee, M. S., et al. (2014). An increase in the Akkermansia spp. population induced by metformin treatment improves glucose homeostasis in diet-induced obese mice. Gut 63, 727-735. doi: 10.1136/gutjnl-2012-303839
Stern, M. P. (1995). Diabetes and cardiovascular disease. The "common soil" hypothesis. Diabetes 44, 369-374. doi: 10.2337/diab.44.4.369

Thaiss, C. A., Levy, M., Korem, T., Dohnalova, L., Shapiro, H., Jaitin, D. A., et al. (2016). Microbiota diurnal rhythmicity programs host transcriptome oscillations. Cell 167, 1495.e12-1510.e12. doi: 10.1016/j.cell.2016.11.003

Tilg, H., Adolph, T. E., Gerner, R. R., and Moschen, A. R. (2018). The intestinal microbiota in colorectal cancer. Cancer Cell 33, 954-964. doi: 10.1016/j.ccell. 2018.03.004

van Passel, M. W., Kant, R., Zoetendal, E. G., Plugge, C. M., Derrien, M., Malfatti, S. A., et al. (2011). The genome of Akkermansia muciniphila, a dedicated intestinal mucin degrader, and its use in exploring intestinal metagenomes. PLoS One 6:e16876. doi: 10.1371/journal.pone.0016876

Wang, Z., Saha, S., Horn, S. V., Thomas, E., Traini, C., Sathe, G., et al. (2018). Gut microbiome differences between metformin- and liraglutidetreated T2DM subjects. Endocrinol. Diabetes Metab. 1:e00009. doi: 10.1002/ edm2.9

Westwellroper, C., Dai, D. L., Soukhatcheva, G., Potter, K. J., Van, R. N., Ehses, J. A., et al. (2011). IL-1 blockade attenuates islet amyloid polypeptide-induced proinflammatory cytokine release and pancreatic islet graft dysfunction. J. Immunol. 187, 2755-2765. doi: 10.4049/jimmunol.1002854

WestwellRoper, C. Y., Ehses, J. A., and Verchere, C. B. (2014). Resident macrophages mediate islet amyloid polypeptide-induced islet IL-1 $\beta$ production and $\beta$-cell dysfunction. Diabetes 63, 1698-1711. doi: 10.2337/db 13-0863

Wu, H., Esteve, E., Tremaroli, V., Khan, M. T., Caesar, R., Mannerås-Holm, L., et al. (2017). Metformin alters the gut microbiome of individuals with treatmentnaive type 2 diabetes, contributing to the therapeutic effects of the drug. Nat. Med. 23, 850-858. doi: 10.1038/nm.4345

Xu, X., Gao, C., Liu, Z., Wu, J., Han, J., Yan, M., et al. (2016). Characterization of the levan produced by Paenibacillus bovis sp. nov BD3526 and its immunological activity. Carbohydr. Polym. 144, 178-186. doi: 10.1016/j.carbpol.2016.02.049

Yu, T. C., Guo, F., Yu, Y., Sun, T., Ma, D., Han, J., et al. (2017). Fusobacterium nucleatum promotes chemoresistance to colorectal cancer by modulating autophagy. Cell 170, 548.e16-563.e16. doi: 10.1016/j.cell.2017.07.008

Zhang, X., Zhao, Y., Xu, J., Xue, Z., Zhang, M., Pang, X., et al. (2015). Modulation of gut microbiota by berberine and metformin during the treatment of high-fat diet-induced obesity in rats. Sci. Rep. 5:14405. doi: 10.1038/srep14405

Zhao, L., Zhang, F., Ding, X., Wu, G., Lam, Y. Y., Wang, X., et al. (2018). Gut bacteria selectively promoted by dietary fibers alleviate type 2 diabetes. Science 359, 1151-1156. doi: 10.1126/science.aao5774

Conflict of Interest Statement: ZQ, JH, HF, CG, JW, CY, ZL, and ZW were employed by Bright Dairy \& Food Co., Ltd.

The remaining authors declare that the research was conducted in the absence of any commercial or financial relationships that could be construed as a potential conflict of interest.

Copyright (C) 2019 Qiao, Han, Feng, Zheng, Wu, Gao, Yang, You, Liu and Wu. This is an open-access article distributed under the terms of the Creative Commons Attribution License (CC BY). The use, distribution or reproduction in other forums is permitted, provided the original author(s) and the copyright owner(s) are credited and that the original publication in this journal is cited, in accordance with accepted academic practice. No use, distribution or reproduction is permitted which does not comply with these terms. 